\title{
Twelve years of Glitches in the Vela Pulsar
}

\author{
Claire S. Flanagan
}

Hartebeesthoek Radio Astronomy Observatory, South Africa

\section{Introduction}

The Vela pulsar (PSR B0833-45) has been observed from Hartebeesthoek RAO since 1984 , as part of an ongoing monitoring project. During this time, the pulsar has undergone five sudden and relatively large spin-ups ("glitches"). Good observational coverage was obtained for the four most recent of these events.

\section{Post-glitch Timing Behaviour}

The post-glitch behaviour of $\dot{\nu}(t)$ is modelled by:

$$
\begin{aligned}
\dot{\nu}\left(t-T_{G}\right)= & \dot{\nu}_{0}+\ddot{\nu}_{0}\left(t-T_{G}\right)+\sum_{i} \Delta \dot{\nu}_{i} \exp \left(-\left(t-T_{G}\right) / \tau_{i}\right) \\
& +\Delta \dot{\nu}_{n l}\left[1-\frac{1}{1+e^{-\left(t-T_{G}\right) / \tau_{n l}}\left(e^{t_{0} / \tau_{n l}}-1\right)}\right],
\end{aligned}
$$

where $T_{G}$ is the glitch epoch and subscript $n l$ refers to a "non-linear" recovery component of the form discussed by Alpar et al. (1989). The results of a preliminary fit of this model to all five glitches are given in table 1 . The "baseline model" describes the long-term behaviour; no single simple model provides an adequate fit to all three of the longer inter-glitch eras observed (Flanagan 1996). The immediate post-glitch behaviour (the "transients" in table 1) are modelled with simple exponentials; three such components are required for the larger and better-observed glitches of 1988 and 1991. Following Alpar et al. (1993), a model incorporating common timescales for all five glitches was also fitted. The data are consistent with a recovery described by at least three exponential decays of $\Delta \dot{\nu}$, over timescales $50 \pm 20 \mathrm{~d}, 5 \pm 2 \mathrm{~d}$ and $0.49 \pm 0.08 \mathrm{~d}$, following each of the most recent five glitches. In addition, the moment of inertia $I_{i}=$ $\Delta \dot{\Omega}_{i} \tau_{i} / \Delta \Omega_{c} I$ associated with the $5 \mathrm{~d}$ component is $(5 \pm 3) \times 10^{-3}$. The data are inconsistent with the $0.49 \mathrm{~d}$ recovery being associated with the same moment of inertia (as defined here) at every glitch. 
Table 1. Model Parameters.

\begin{tabular}{|c|c|c|c|c|c|c|}
\hline & & 1985 & 1988 & 1991 & 1994 & $1994 \mathrm{~A}$ \\
\hline & & $-155.54(3)$ & $-156.45(3)$ & $-156.62(4)$ & $-156.54(3)$ & $-156.59(3)$ \\
\hline $\begin{array}{l}\ddot{\nu}_{0} \\
10^{-22} \mathrm{~Hz} \mathrm{~s}^{-2}\end{array}$ & & & $+9.3(7)$ & $+8.3(2)$ & & \\
\hline$\Delta \dot{\nu}_{n l} / \dot{\nu}$ & $\%$ & & $+0.11(2)$ & & & \\
\hline & days & & $9(6)$ & & & \\
\hline & days & & $468(6)$ & & & \\
\hline$\Delta \dot{\nu} / \dot{\nu}$ & $\%$ & $+0.87(2)$ & & & & \\
\hline$\tau$ & days & $760(40)$ & & & & \\
\hline \multicolumn{7}{|l|}{ transients: } \\
\hline$\Delta \dot{\nu}_{1} / \dot{\nu}$ & $\%$ & & $+0.26(4)$ & $+0.35(6)$ & & \\
\hline$\tau_{1}$ & days & & $70(20)$ & $49(6)$ & & \\
\hline$\Delta \dot{\nu}_{2} / \dot{\nu}$ & $\%$ & $+1.8(3)$ & $+1.2(5)$ & $+2.3(7)$ & & $+0.4(2)$ \\
\hline$\tau_{2}$ & days & $6.8(4)$ & $5(1)$ & $4.9(6)$ & & $6(7)$ \\
\hline $\begin{array}{l}\Delta \dot{\nu}_{3} / \dot{\nu} \\
\tau_{3}\end{array}$ & $\%$ & & $+16(6)$ & $+40(10)$ & & \\
\hline$\tau_{3}$ & days & & $0.53(9)$ & $0.59(4)$ & & \\
\hline
\end{tabular}

The components listed in column 1 are those of eqn. 1.

$T_{G}$ is the glitch epoch.

Errors are $2-\sigma$ in the least significant digit quoted.

\section{References}

Alpar, M. A., Cheng, K. S. \& Pines, D. 1989, ApJ, 346, 823

Alpar, M. A., Chau, H. F., Cheng, K. S. \& Pines, D. 1993, ApJ, 409, 345

Flanagan, C. S. 1996, in preparation 